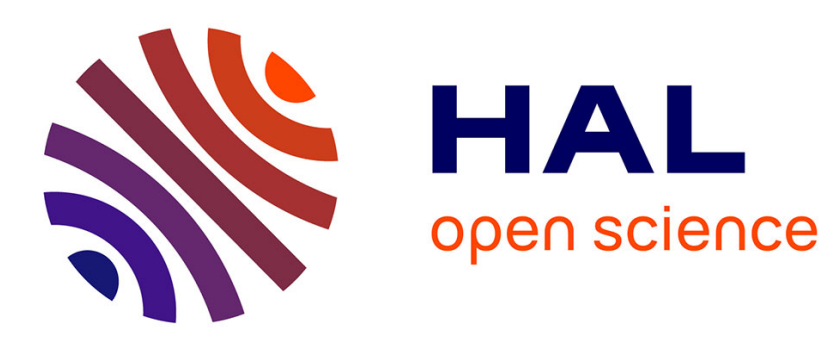

\title{
Internal controllability of first order quasilinear hyperbolic systems with a reduced number of controls
}

Fatiha Alabau-Boussouira, Jean-Michel Coron, Guillaume Olive

\section{To cite this version:}

Fatiha Alabau-Boussouira, Jean-Michel Coron, Guillaume Olive. Internal controllability of first order quasilinear hyperbolic systems with a reduced number of controls. SIAM Journal on Control and Optimization, 2017, 55 (1), pp.300-323. hal-01139980

\section{HAL Id: hal-01139980 \\ https://hal.science/hal-01139980}

Submitted on 7 Apr 2015

HAL is a multi-disciplinary open access archive for the deposit and dissemination of scientific research documents, whether they are published or not. The documents may come from teaching and research institutions in France or abroad, or from public or private research centers.
L'archive ouverte pluridisciplinaire HAL, est destinée au dépôt et à la diffusion de documents scientifiques de niveau recherche, publiés ou non, émanant des établissements d'enseignement et de recherche français ou étrangers, des laboratoires publics ou privés. 


\title{
INTERNAL CONTROLLABILITY OF FIRST ORDER QUASILINEAR HYPERBOLIC SYSTEMS WITH A REDUCED NUMBER OF CONTROLS
}

\author{
FATIHA ALABAU-BOUSSOUIRA, JEAN-MICHEL CORON AND GUILLAUME OLIVE*
}

\begin{abstract}
In this paper we investigate the exact controllability of $n \times n$ first order quasilinear hyperbolic systems by $m<n$ internal controls that are localized in space in some part of the domain. We distinguish two situations. The first one is when the equations of the system have the same speed. In this case, we can use the method of characteristics and obtain a simple and complete characterization for linear systems. Thanks to a linear test this also provides some sufficient conditions for the local exact controllability around the trajectories of semilinear systems. However, when the speed of the equations are not anymore the same, we see that we encounter the problem of loss of derivatives if we try to control quasilinear systems with a reduced number of controls. To solve this problem, as in a prior article by J.-M. Coron and P. Lissy on a Navier-Stokes control system, we first use the notion of algebraic solvability due M. Gromov. However, in contrast with this prior article where a standard fixed point argument could be used to treat the nonlinearities, we use here a fixed point theorem of Nash-Moser type due to M. Gromov in order to handle the problem of loss of derivatives.
\end{abstract}

Key words. Quasilinear hyperbolic systems, exact internal controllability, controllability of systems, algebraic solvability.

AMS subject classifications. 35L50, 93B05, 93C10.

1. Introduction. In this paper we investigate the exact controllability of $n \times$ $n$ first order quasilinear hyperbolic systems by $m<n$ internal controls that are localized in space in some part of the domain. While the controllability of quasilinear hyperbolic systems by boundary controls has been intensively studied, Cir69, LR02, LR03, Wan06, Zha09, LRW10, to our knowledge there are no equivalent results for the internal controllability. On the other hand, the controllability of systems of PDEs with a reduced number of controls has been a challenging problem for the last decades, see for instance AB03, ABL12, AB13, DLRL14, AB14 for linear hyperbolic systems and [ha09] for quasilinear hyperbolic systems, BGBPG02, GBPG05, Gue07] and the survey AKBGBdT11 for linear parabolic systems, CGR10, CL14 for nonlinear parabolic systems, CG09b for Stokes equations, FCGIP06, CG09a and CL14 for Navier-Stokes equations. Let us also point out that, in many of these articles, the general strategy is to start with a controllability result in the case where there are as many controls as the number of equations and then to try to remove some of these controls by a suitable procedure. We also follow this general strategy here.

In [LR03, the authors introduced a constructive method to control quasilinear systems of $n$ equations by $n$ boundary controls. This proficient method is based on existence and uniqueness results of semi-global solutions [LJ01] (i.e. with large time and small data) that they apply to several mixed initial-boundary value problems, using also the equivalent roles of the time and the space. As we shall see below, using a method of extension of the domain (as it is often used in the parabolic framework), we can recover this result for the internal controllability, that is we can prove the controllability of $n \times n$ quasilinear systems by $n$ internal controls. The situation is more complicated when we have less controls than equations. Indeed, even though some results are known for the boundary controllability of $n \times n$ quasilinear systems

${ }^{*}$ Jean-Michel Coron and Guillaume Olive were supported by the ERC advanced grant 266907 (CPDENL) of the 7th Research Framework Programme (FP7) 
with $m<n$ controls [Zha09], the extension method is not anymore applicable in this context. Thus, we need to develop direct methods to solve the problem of internal controllability.

We start the study with linear systems of equations with the same velocity. In this case, we can apply the method of characteristics and obtain a complete and simple characterization of the exact controllability. We show that the linear system can be viewed as a parametrized family of ODEs that are controlled independently. The difficulty is actually to prove that this is enough to build a smooth $\left(C^{1}\right)$ control for the linear hyperbolic system. Moreover, since we look for controls of the hyperbolic system that are localized in some part of the domain, a nonstandard condition on the supports of the ODEs also appears and needs to be handled. Another key point of the proof is the explicit formula of the HUM control for ODEs.

Using then a standard fixed point argument we can obtain sufficient conditions for the local exact controllability around the trajectories of semilinear systems. However, when the equations do not have the same speed anymore and the nonlinearity is stronger, that is when we consider quasilinear systems, the standard linear test fails because of a loss of derivatives. To solve this problem, we need to use a fixed point of Nash-Moser type. We propose to use the fixed point theorem of M. Gromov Gro86, Section 2.3.2, Main Theorem], which is based on the notion of algebraic solvability for partial differential operators. The method consists in, first controlling the $n \times n$ system by $n$ controls, and then to eliminate a certain number of controls through the algebraic solvability. The use of the Gromov algebraic solvability in the framework of the control theory was introduced in Cor07, Pages 13-15] in the framework of the control of linear ordinary differential equations (however it does not lead to new results in this case) and in CL14 for a for a Navier-Stokes control system. In this last case, the parabolicity allows to have smooth controls, as shown in [CL14, and thus to avoid the problem of loss of derivatives. The difference between the present work and CL14, where the algebraic solvability was the difficult task (the fixed point was standard), is that, following the algebraic solvability step, we show how to apply the fixed point theorem of Gromov to obtain the controllability of the quasilinear system. Last, but not least, this method is probably not optimal with the regularity obtained, which leaves some challenging problems.

\section{Systems of equations with the same velocity.}

2.1. Linear systems. Let us consider the following linear hyperbolic system with periodic boundary conditions:

$$
\left\{\begin{array}{l}
y_{t}+y_{x}+A(t, x) y=B(t, x) \Theta, \quad(t, x) \in[0, T] \times[0, L], \\
y(t, L)=y(t, 0), \quad t \in[0, T], \\
y(0, x)=y^{0}(x), \quad x \in[0, L] .
\end{array}\right.
$$

In (2.1), $T>0$ is the control time, $L>0$ is the length of the domain. $A$ and $B$ are time and space dependent matrices of size $n \times n$ and $n \times m$, respectively, where $n \in \mathbb{N}^{*}$ denotes the number of equations of the system and $m \in \mathbb{N}^{*}$ the number of controls (with possibly $m<n$ ). $y^{0}$ is the initial data and $y(t, \cdot):[0, L] \longrightarrow \mathbb{R}^{n}$ is the state at time $t \in[0, T]$. Finally, $\Theta(t, \cdot):[0, L] \longrightarrow \mathbb{R}^{m}$ is the distributed control at time $t \in[0, T]$, that we look subject to the constraint

$$
\operatorname{supp} \Theta \subset[0, T] \times[a, b],
$$


where here, and in what follows, the interval $[a, b]$, with $0 \leq a<b \leq L$, is fixed.

Throughout this article, for $k \in \mathbb{N}$ and $p \in \mathbb{N}^{*}$, we denote by $C_{L}^{k}([0, T] \times[0, L])^{p}$ $\left(\right.$ resp. $\left.C_{L}^{k}([0, L])^{p}\right)$ the Banach space of functions $y \in C_{L}^{k}([0, T] \times[0, L])^{p}($ resp. $y \in$ $\left.C_{L}^{k}([0, L])^{p}\right)$ that are $x L$-periodic, that is

$$
\begin{gathered}
\partial_{x}^{i} y(t, 0)=\partial_{x}^{i} y(t, L), \quad \forall t \in[0, T], \quad \forall i \in \llbracket 0, k \rrbracket . \\
\left(\text { resp. } y^{(i)}(0)=y^{(i)}(L), \quad \forall i \in \llbracket 0, k \rrbracket .\right)
\end{gathered}
$$

All along Section 2.1] we assume that $A \in C_{L}^{1}([0, T] \times[0, L])^{n \times n}, B \in C_{L}^{1}([0, T] \times$ $[0, L])^{n \times m}$. These assumptions are made for regularity purposes, see below.

We recall that, for every $T>0$, there exists $C>0$ such that, for every $\Theta \in$ $C_{L}^{1}([0, T] \times[0, L])^{m}$ and every $y^{0} \in C_{L}^{1}([0, L])^{n}$, there exists a unique classical global solution $y \in C_{L}^{1}([0, T] \times[0, L])^{n}$ to (2.1) , and this solution satisfies the estimate

$$
\|y\|_{C^{1}} \leq C\left(\left\|y^{0}\right\|_{C^{1}}+\|\Theta\|_{C^{1}}\right) .
$$

This well-posedness result follows from the classical theory of linear hyperbolic systems using the method of characteristics [LY85. Note that the kind of boundary conditions we consider is nonlocal but, as already noticed in [CBdN08] (see also [LRW10]), it can always be reduced to more standard (i.e. local) boundary conditions by introducing the enlarged system satisfied by $(y, \tilde{y})$ where $\tilde{y}(t, x)=y(t, L-x)$.

DEFINITION 2.1. We say that System (2.1) is exactly controllable at time $T>0$ if, for every $y^{0} \in C_{L}^{1}([0, L])^{n}$ and for every $y^{1} \in C_{L}^{1}([0, L])^{n}$, there exists a control $\Theta \in$ $C_{L}^{1}([0, T] \times[0, L])^{m}$ that satisfies the constraint (2.2) and is such that the corresponding solution $y \in C_{L}^{1}([0, T] \times[0, L])^{n}$ to (2.1) satisfies

$$
y(T, x)=y^{1}(x), \quad \forall x \in[0, L] .
$$

2.1.1. The extended characteristics. Let us now introduce an important tool when dealing with hyperbolic systems, namely the characteristics of the system. In our case (speed 1 on each equation), the characteristic $X$ of System (2.1) passing through the point $\left(t_{0}, x_{0}\right) \in[0, T] \times[0, L]$ is the straight line

$$
X\left(t, t_{0}, x_{0}\right) \stackrel{\text { def }}{=} t-t_{0}+x_{0}, \quad t \in[0, T] .
$$

However, in this paper, the crucial tools we need are the extended characteristics $\bar{X}:[0, T] \times[0, L) \longrightarrow[0, L]$, defined by (see Fig. 2.1] below):

$$
\bar{X}(t, x) \stackrel{\text { def }}{=} \begin{cases}X(t, 0, x) & \text { if } t \in\left[0, \tau_{0}(x, L)\right], \\ X\left(t, \tau_{k-1}(x, L), 0\right) & \text { if } t \in\left(\tau_{k}(x, 0), \tau_{k}(x, L)\right], \quad k \in \llbracket 1, k_{\max }(x, 0) \rrbracket,\end{cases}
$$

where, for every $k \in \mathbb{N}$ and $c \in[0, L]$, we introduce the functions

$$
\tau_{k}(x, c) \stackrel{\text { def }}{=}\left\{\begin{array}{cl}
0 & \text { if } c-x+k L \in(-\infty, 0), \\
c-x+k L & \text { if } c-x+k L \in[0, T], \\
T & \text { if } c-x+k L \in(T,+\infty),
\end{array}\right.
$$


and $k_{\min }(x, c)$ (resp. $\left.k_{\max }(x, c)\right)$ denotes the smallest (resp. greatest) integer $k \in \mathbb{N}$ such that $c-x+k L>0($ resp. $c-x+k L<T)$. More precisely, denoting by $\lfloor\cdot\rfloor$ the floor function and $\lceil\cdot\rceil$ the ceiling function,

$$
\begin{gathered}
k_{\min }(x, c) \stackrel{\text { def }}{=}\left\lfloor\frac{-c+x}{L}\right\rfloor+1=\left\{\begin{array}{cc}
0 & \text { if } x \in[0, c), \\
1 & \text { if } x \in[c, L),
\end{array}\right. \\
k_{\text {max }}(x, c) \stackrel{\text { def }}{=}\left\lceil\frac{T-c+x}{L}\right\rceil-1=\left\{\begin{array}{cc}
\left\lceil\frac{T-c}{L}\right\rceil-1 & \text { if } x \in[0, p(c)], \\
\left\lceil\frac{T-c}{L}\right\rceil & \text { if } x \in(p(c), L),
\end{array}\right.
\end{gathered}
$$

where

$$
p(c) \stackrel{\text { def }}{=}\left(\left\lceil\frac{T-c}{L}\right\rceil-\frac{T-c}{L}\right) L .
$$

Note that $\tau_{k}(x, 0)=\tau_{k-1}(x, L)$ for every $k \geq 1$ and $\tau_{k_{\max }(x, 0)}(x, L)=T$, so that $\bar{X}(t, x)$ is indeed defined for every $t \in[0, T]$.

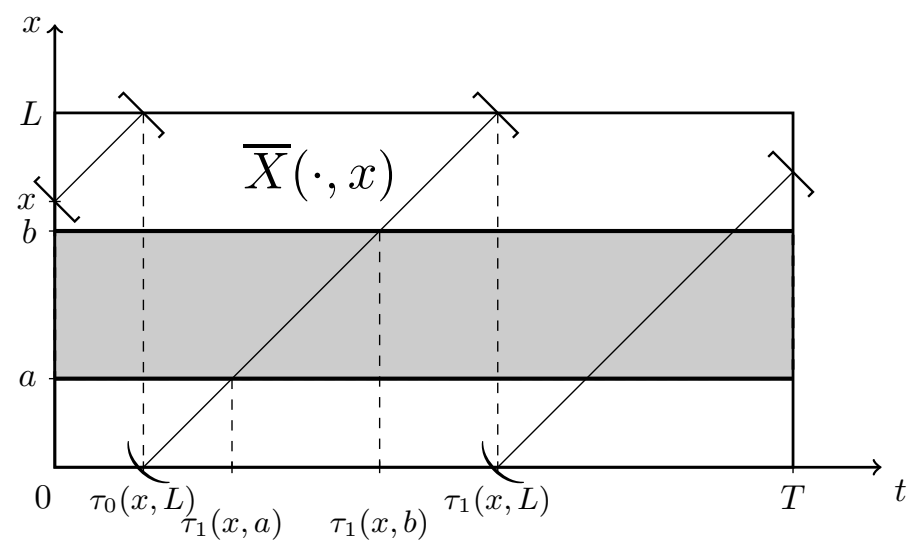

Fig. 2.1: The extended characteristics $\bar{X}(\cdot, x)$.

For $0 \leq a<b \leq L$, we list below some properties of these functions, under the essential assumption that every extended characteristic $\bar{X}$ crosses the domain $[0, T] \times[a, b]$ at some time, that is

$$
T>L-(b-a) .
$$

1. $k_{\min }(x, b) \leq k_{\max }(x, a)$.

2. $\tau_{k}(x, a)<\tau_{k}(x, b)$ for every $k \in \llbracket k_{\min }(x, b), k_{\max }(x, a) \rrbracket$ if $x \neq b$ and $x \neq p(a)$.

3. $\tau_{k}(x, b) \leq \tau_{k+1}(x, a)$ for every $k \in \llbracket k_{\min }(x, b), k_{\max }(x, a)-1 \rrbracket$.

4. $\bar{X}(t, x) \in(a, b)$ for every $t \in\left(\tau_{k}(x, a), \tau_{k}(x, b)\right)$ for every $k$ satisfying

$$
k \in \llbracket k_{\min }(x, b), k_{\max }(x, a) \rrbracket .
$$


We then introduce the following open sets (see Fig. 2.2 below)

$$
\begin{gathered}
\mathcal{T}_{0} \stackrel{\text { def }}{=}\left\{(t, x) \in(0, T) \times(0, L) \quad \mid \quad t \in\left(0, \tau_{0}(x, L)\right)\right\}, \\
\mathcal{T}_{\left\lceil\frac{T}{L}\right\rceil} \stackrel{\text { def }}{=}\left\{(t, x) \in(0, T) \times(p(0), L), \quad \mid \quad t \in\left(\tau_{\left\lceil\frac{T}{L}\right\rceil}(x, 0), T\right)\right\},
\end{gathered}
$$

and, for $k \in \llbracket 1,\left\lceil\frac{T}{L}\right\rceil-1 \rrbracket$,

$$
\mathcal{T}_{k} \stackrel{\text { def }}{=}\left\{(t, x) \in(0, T) \times(0, L) \quad \mid \quad t \in\left(\tau_{k}(x, 0), \tau_{k}(x, L)\right)\right\} .
$$

We set

$$
\mathcal{T} \stackrel{\text { def }}{=} \bigcup_{k=0}^{\left\lceil\frac{T}{L}\right\rceil} \mathcal{T}_{k}
$$

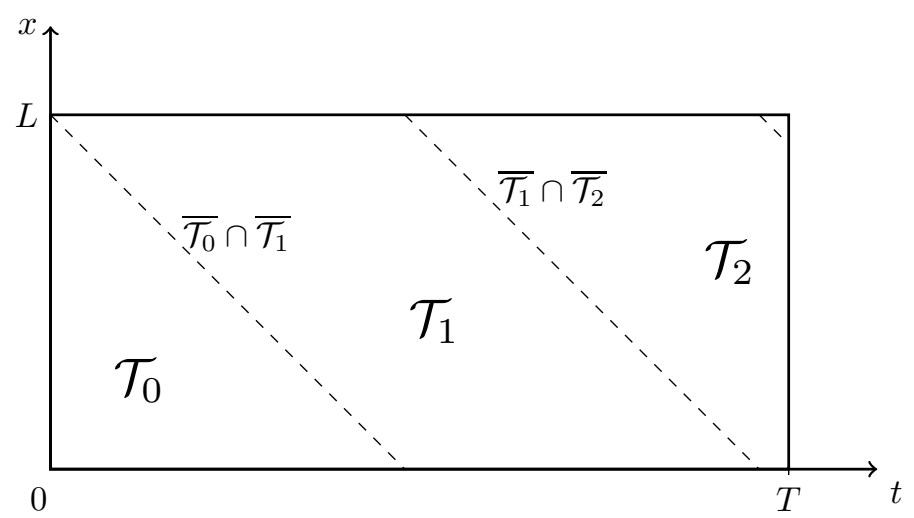

Fig. 2.2: Domains $\mathcal{T}_{k}$ and the parts $\overline{\mathcal{T}_{k}} \cap \overline{\mathcal{T}}_{k+1}$ of their boundary.

REMARK 1. Let us give some comments about the properties of the extended characteristics. First, we have

$$
\bar{X} \in C^{1}(\mathcal{T})
$$

with $\bar{X}_{t}(t, x)=\bar{X}_{x}(t, x)=1$ for every $(t, x) \in \mathcal{T}$. Moreover, at the boundary $\partial \mathcal{T}$, we have

$$
\forall\left(t_{0}, x_{0}\right) \in \overline{\mathcal{T}_{k}} \cap \overline{\mathcal{T}}_{k+1}, \quad \lim _{\substack{(t, x) \rightarrow\left(t_{0}, x_{0}\right) \\(t, x) \in \mathcal{T}_{k}}} \bar{X}(t, x)=L, \quad \lim _{\substack{(t, x) \rightarrow\left(t_{0}, x_{0}\right) \\(t, x) \in \mathcal{T}_{k+1}}} \bar{X}(t, x)=0,
$$

$\lim _{(t, x) \rightarrow\left(t_{0}, 0\right)} \bar{X}(t, x)=\bar{X}\left(t_{0}, 0\right)$ for every $t_{0} \in[0, T]$, and

$$
\forall t_{0} \in[0, T], \quad \lim _{(t, x) \rightarrow\left(t_{0}, L\right)} \bar{X}(t, x)=\left\{\begin{array}{cl}
\bar{X}\left(t_{0}, 0\right) & \text { if } t_{0} \in(0, T] \\
L & \text { if } t_{0}=0 .
\end{array}\right.
$$


In particular $\bar{X}$ has a continuous extension (still denoted by $\bar{X}$ ) to all the points of the boundary $(t, L), t \in[0, T]$.

Second, the map $(t, x) \mapsto(t, \bar{X}(t, x))$ is a $C^{1}$-diffeomorphism from $\mathcal{T}$ to $\mathcal{T}^{\prime}=$ $(0, T) \times(0, L) \backslash\{(t, \bar{X}(t, 0)) \mid t \in[0, T]\}$. We denote by $\left(t, \bar{X}^{-1}(t, x)\right)$ its inverse.

Finally, for every $x \in[0, L]$, we denote by $A_{x}$ and $B_{x}$ the values of $A$ and $B$ along the extended characteristic $\bar{X}(\cdot, x)$ :

$$
A_{x}(t) \stackrel{\text { def }}{=} A(t, \bar{X}(t, x)), \quad B_{x}(t) \stackrel{\text { def }}{=} B(t, \bar{X}(t, x)), \quad \forall t \in[0, T] .
$$

Clearly, $(t, x) \mapsto A_{x}(t) \in C^{1}(\mathcal{T})^{n \times n}$. Since $A$ is $x L$-periodic, we have $A \in$ $C^{0}(\overline{\mathcal{T}})^{n \times n}=C^{0}([0, T] \times[0, L])^{n \times n}$. On the same manner, since $\partial_{t} A$ and $\partial_{x} A$ are $x$ $L$-periodic, we have $A \in C^{1}([0, T] \times[0, L])^{n \times n}$. Note also that $A_{L}=A_{0}$. The same statements hold for the map $(t, x) \mapsto B_{x}(t)$ as well.

2.1.2. Characterization of the controllability of (2.1). The main result of section 2.1 is the following:

Theorem 2.2. Let $T, L>0$ and $0 \leq a<b \leq L$. System (2.1) is exactly controllable at time $T$ if, and only if, the following 2 conditions hold:

( $\left.\mathcal{H}_{1}\right) T>L-(b-a)$.

$\left(\mathcal{H}_{2}\right)$ For every $x \in[0, L)$, the following $O D E$ is controllable:

$$
\left\{\begin{array}{l}
\frac{d}{d t} z(t)=-A_{x}(t) z(t)+B_{x}(t) \psi(t), \quad \forall t \in[0, T] \\
z(0)=z^{0} \in \mathbb{R}^{n}
\end{array}\right.
$$

with controls $\psi$ such that

$$
\psi \equiv 0 \text { in }[0, T] \backslash\left(\bigcup_{k=k_{\min }(x, b)}^{k_{\max }(x, a)}\left[\tau_{k}(x, a), \tau_{k}(x, b)\right]\right) .
$$

Remark 2. When $[a, b]=[0, L]$, hypothesis $\left(\mathcal{H}_{1}\right)$ and (2.5) are automatically satisfied.

Remark 3. As we shall see below (Proposition 2.3) the controllability of (2.4) with (2.5) only depends on the values of $A$ and $B$ inside the control domain $[0, T] \times$ $[a, b]$.

REMARK 4. In the proof of Theorem 2.2 we will explicitly construct a control $\Theta$ that steers the solution $y$ to (2.1) from $y^{0}$ to $y^{1}$, see (2.14) below. We can see that this control $\Theta$ satisfies the following additional properties:

1. Continuity: there exists $C>0$ (depending only on $T, L, a, b, A, B$ ) such that

$$
\|\Theta\|_{C^{1}} \leq C\left(\left\|y^{0}\right\|_{C^{1}}+\left\|y^{1}\right\|_{C^{1}}\right) .
$$

2. Locality: there exists $\delta>0$ small enough (depending only on $T, L, a, b, A, B$ ), such that

$$
\operatorname{supp} \Theta \subset[\delta, T-\delta] \times[a+\delta, b-\delta] .
$$

3. Higher regularity: if $y^{0}, y^{1} \in C_{L}^{k}([0, L])^{n}$ and $A \in C_{L}^{k}([0, T] \times[0, L])^{n \times n}$, $B \in C_{L}^{k}([0, T] \times[0, L])^{m \times n}(k \geq 1)$, then

$$
\Theta \in C^{k}([0, T] \times[0, L])^{m} .
$$


2.1.3. Controllability of linear O.D.E with constraints. Let us recall that we know some powerful tools to characterize the controllability of linear time-varying ODEs if no constraint are imposed on the controls. We state below the extensions of these theorems to the case where the controls are supported in some part of the domain.

Let us consider the $n \times n$ ODE

$$
\left\{\begin{array}{l}
\frac{d}{d t} z(t)=-A(t) z(t)+B(t) \psi(t), \quad \forall t \in[0, T], \\
z(0)=z^{0} \in \mathbb{R}^{n}
\end{array}\right.
$$

with $A \in C^{1}([0, T])^{n \times n}, B \in C^{1}([0, T])^{n \times m}$. We want to characterize the controllability of (2.7) with the following additional constraint on the controls:

$$
\psi \equiv 0 \text { in }[0, T] \backslash\left(\bigcup_{i=1}^{M}\left[a_{i}, b_{i}\right]\right),
$$

where $0 \leq a_{i}<b_{i} \leq T$ are such that $b_{i} \leq a_{i+1}$ for every $i \in \llbracket 1, M-1 \rrbracket$.

Let us denote by $R \in C^{1}([0, T] \times[0, T])^{n \times n}$ the resolvent associated with $-A \in$ $C^{1}([0, T])^{n \times n}$, that is, for every $s \in[0, T], R(\cdot, s)$ is the classical solution to the ODE

$$
\left\{\begin{array}{l}
\partial_{t} R(t, s)=-A(t) R(t, s), \quad \forall t \in[0, T], \\
R(s, s)=\mathrm{Id} .
\end{array}\right.
$$

Proposition 2.3. The ODE (2.7) is controllable with (2.8) if, and only if, its controllability Gramian, that is the $n \times n$ matrix

$$
Q \stackrel{\text { def }}{=} \sum_{i=1}^{M} \int_{a_{i}}^{b_{i}} R(T, s) B(s) B(s)^{*} R(T, s)^{*} d s,
$$

is invertible.

The proof of Proposition 2.3 can be adapted from the one of [KHN63, Theorem 5]. To do so, we consider the control problem (2.7) with $\eta B$ instead of $B$, where $\eta$ is a cutoff function that vanishes outside $\bigcup_{i=1}^{M}\left[a_{i}, b_{i}\right]$ and is equal to 1 in $\bigcup_{i=1}^{M}\left[a_{i}+\varepsilon, b_{i}-\varepsilon\right]$ with $\varepsilon>0$ small enough so that, by continuity, the Gramian (2.9) with $a_{i}+\varepsilon$ (resp. $\left.b_{i}-\varepsilon\right)$ instead of $a_{i}\left(\right.$ resp. $\left.b_{i}\right)$ remains invertible.

Thanks to the previous characterization, we obtain the following propostions (see [KHN63, Theorem 10] and [SM67] for a proof)

Proposition 2.4. Assume that $A$ and $B$ are constant. Then, the controllability of (2.7)-(2.8) is equivalent to the algebraic condition

$$
\operatorname{rank}[A: B]=n,
$$

where the $n \times n m$ matrix $[A: B]$ is defined by

$$
[A: B] \stackrel{\text { def }}{=}\left[B|A B| A^{2} B|\cdots| A^{n-1} B\right] .
$$

Proposition 2.5. Assume that

$$
A \in C^{n-2}([0, T])^{n \times n} \text { and } B \in C^{n-1}([0, T])^{n \times m}
$$


and let us introduce the following notation:

$$
\forall t \in[0, T], \quad\left\{\begin{array}{l}
B_{0}(t)=B(t), \\
B_{j}(t)=\frac{d}{d t} B_{j-1}(t)+A(t) B_{j-1}(t), \quad \forall j \in \llbracket 1, n-1 \rrbracket,
\end{array}\right.
$$

and, for every $t \in[0, T]$, the $n \times n m$ matrix

$$
[A: B](t) \stackrel{\text { def }}{=}\left[B_{0}(t)\left|B_{1}(t)\right| \cdots \mid B_{n-1}(t)\right]
$$

(which provides an extension of (2.10)). Then, the ODE (2.7) is controllable with (2.8) if the following property holds:

$$
\exists i \in \llbracket 1, M \rrbracket, \quad \exists t_{i} \in\left[a_{i}, b_{i}\right], \quad \operatorname{rank}[A: B]\left(t_{i}\right)=n .
$$

2.1.4. Proof of Theorem 2.2, sufficient part. The proof of the sufficient part of Theorem 2.2 relies on the following key lemma (the proof of which is postponed to the appendix; see Section $\mathrm{A}$. It states that we can always reduce a little bit the domain of control. This is a uniform result with respect to $x$ (compare with Proposition 2.3).

All along this section, for $x \in[0, L]$ we denote by $R_{x} \in C^{1}([0, T] \times[0, T])^{n \times n}$ the resolvent associated with $-A_{x} \in C^{1}([0, T])^{n \times n}$. It is important to notice that the map $(t, s, x) \mapsto R_{x}(t, s)$ is of class $C^{1}([0, T] \times[0, T] \times[0, L])^{n \times n}$ since the map $(t, x) \mapsto A_{x}(t)$ is also of class $C^{1}([0, T] \times[0, L])^{n \times n}$ (see, for instance Har82, Chapter $\mathrm{V}$, Theorem 3.1]).

Lemma 2.6. Assume that $\left[\mathcal{H}_{1}\right)$ and $\left[\left(\mathcal{H}_{2}\right)\right.$ hold. Then, there exists $\delta>0$ small enough and a cut-off function $\eta \in C^{1}([0, T] \times[0, L])$ with (see Fig. [2.3)

$$
\eta \equiv 0 \text { in }[0, T] \times[0, L] \backslash((\delta, T-\delta) \times(a+\delta, b-\delta)),
$$

such that, for every $x \in[0, L]$, the Gramian

$$
Q_{x} \stackrel{\text { def }}{=} \int_{0}^{T} R_{x}(T, s) B_{x}(s) B_{x}(s)^{*} R_{x}(T, s)^{*} \eta(s, \bar{X}(s, x)) d s,
$$

is invertible.

Proof of Theorem 2.2 (sufficient part). Assume that $\left[\mathcal{H}_{1}\right)$ and $\left.\left(\mathcal{H}_{2}\right)\right]$ hold and let us show that System (2.1) is exactly controllable at time $T$. Let $y^{0}, y^{1} \in C_{L}^{1}([0, L])^{n}$.

Let $Q_{x}$ be the controllability Gramian defined by (2.12). For every $(t, x) \in[0, T] \times$ $[0, L]$, we set

$$
\psi(t, x) \stackrel{\text { def }}{=} \eta(t, \bar{X}(t, x)) B_{x}(t)^{*} R_{x}(T, t)^{*} Q_{x}^{-1}\left(y^{1}(\bar{X}(T, x))-R_{x}(T, 0) y^{0}(x)\right) .
$$

Since $\eta \in C_{L}^{1}([0, T] \times[0, L])$, we have $(t, x) \mapsto \eta(t, \bar{X}(t, x)) \in C^{1}([0, T] \times[0, L])$. Using Lebesgue's dominated convergence theorem, we obtain $x \mapsto Q_{x} \in C^{1}([0, L])^{n \times n}$. On the other hand, since $y^{1} \in C_{L}^{1}([0, L])^{n}$, we have $x \mapsto y^{1}(\bar{X}(T, x)) \in C^{1}([0, L])^{n}$. As a result,

$$
\psi \in C^{1}([0, T] \times[0, L])^{m}
$$




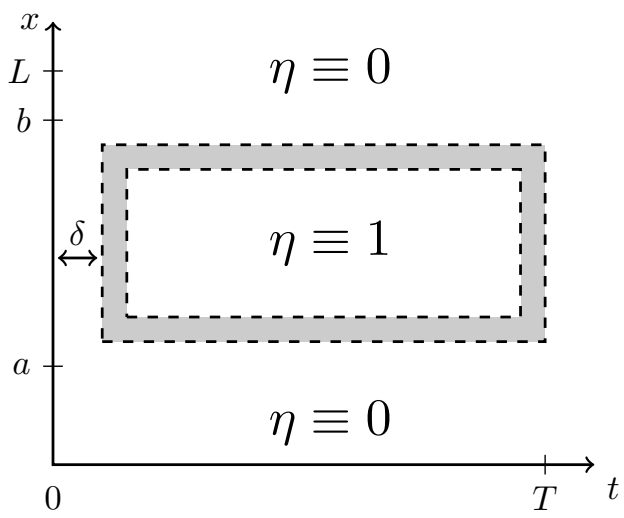

Fig. 2.3: Reduction of the control domain.

For every $(t, x) \in[0, T] \times[0, L]$, we set

$$
\Theta(t, x) \stackrel{\text { def }}{=}\left\{\begin{array}{cl}
\psi\left(t, \bar{X}^{-1}(t, x)\right) & \text { if }(t, x) \in \mathcal{T}^{\prime}, \\
\psi(t, 0) & \text { if } x=\bar{X}(t, 0), \\
0 & \text { if }(t, x) \in \partial([0, T] \times[0, L]) .
\end{array}\right.
$$

From (2.11) we have $\Theta \in C^{1}([0, T] \times[0, L])^{m}$ and (2.6). Moreover,

$$
\Theta(t, \bar{X}(t, x))=\psi(t, x), \quad \forall(t, x) \in[0, T] \times[0, L] .
$$

Let $y \in C^{1}([0, T] \times[0, L])^{m}$ be the solution to (2.1) associated with $\Theta$ defined by (2.14). For every $x \in[0, L)$, writing $y$ along the extended characteristics $\bar{X}(\cdot, x)$, we see that $t \mapsto y(t, \bar{X}(t, x))$ solves the ODE (2.4) with $\psi$ defined by (2.13) and $z^{0}=y^{0}(x)$ (at least in the weak sense $\left.W^{1, \infty}(0, T)^{n}\right)$. As a result we obtain $y(T, \bar{X}(T, x))=y^{1}(\bar{X}(T, x))$ for every $x \in[0, L)$. Since $x \mapsto \bar{X}(T, x)$ defines a bijective map from $[0, L)$ to $(0, L]$, we obtain that $y(T, x)=y^{1}(x)$ for every $x \in[0, L)$. By continuity it follows that $y(T, x)=y^{1}(x)$ for every $x \in[0, L]$.

2.1.5. Proof of Theorem 2.2, necessary part. Assume now that System (2.1) is exactly controllable at time $T$ and let us prove that this implies that $\left(\mathcal{H}_{1}\right)$ and $\left(\mathcal{H}_{2}\right)$ hold.

Assume first that $0<T \leq L-(b-a)$ and let $t_{0}=\max (0, T-a)$ and $x_{0}=-t_{0}+L$. Note that $t_{0} \in[0, T]$ and $x_{0} \in[0, L]$. Let $y^{1}=0$ and let $y^{0} \in C_{L}^{1}([0, L])^{n}$ be such that

$$
y^{0}\left(x_{0}\right) \neq 0 .
$$

Writing $y$ along the characteristic $X\left(s, t_{0}, 0\right)$ for $s \in\left[t_{0}, T\right]$, gives

$$
y\left(T, X\left(T, t_{0}, 0\right)\right)=R_{1}\left(T, t_{0}\right) y\left(t_{0}, 0\right)+\int_{t_{0}}^{T} R_{1}(T, s) B(s) \Theta\left(s, X\left(s, t_{0}, 0\right)\right) d s,
$$

where $R_{1}$ is the resolvent associated with $t \in\left[t_{0}, T\right] \mapsto A\left(t, X\left(t, t_{0}, 0\right)\right)$. Now observe that, since $T \leq t_{0}+a$, we have $X\left(s, t_{0}, 0\right) \leq a$ for $s \in\left[t_{0}, T\right]$, so that, thanks to (2.2),

$$
\Theta\left(s, X\left(s, t_{0}, 0\right)\right)=0, \quad \forall s \in\left[t_{0}, T\right] .
$$


As a result,

$$
y\left(T, X\left(T, t_{0}, 0\right)\right)=R_{1}\left(T, t_{0}\right) y\left(t_{0}, 0\right) .
$$

Similarly, writing $y$ along the characteristic $X\left(s, 0, x_{0}\right)$ for $s \in\left[0, t_{0}\right]$, and using that $x_{0} \geq b$, this leads to

$$
y\left(t_{0}, L\right)=y\left(t_{0}, X\left(t_{0}, 0, x_{0}\right)\right)=R_{2}\left(t_{0}, 0\right) y^{0}\left(x_{0}\right),
$$

where $R_{2}$ is the resolvent associated with $t \in\left[0, t_{0}\right] \mapsto A\left(t, X\left(t, 0, x_{0}\right)\right)$. Since $y\left(t_{0}, 0\right)=y\left(t_{0}, L\right)$, the previous equality can be combined with (2.16) and (2.15) to show that $y\left(T, X\left(T, t_{0}, 0\right)\right) \neq 0$ and therefore System (2.1) is not exactly controllable at time $T$.

We turn out to the necessity of $\left(\mathcal{H}_{2}\right)$. Let $z^{0}, z^{1} \in \mathbb{R}^{n}$ be fixed. We then define $y^{0} \equiv z^{0}$ and $y^{1} \equiv z^{1}$, which belong to $C_{L}^{1}([0, L])^{n}$. Thus, by assumption, there exists $\Theta \in C_{L}^{1}([0, T] \times[0, L])^{m}$ that satisfies (2.2) such that the corresponding solution $y \in C^{1}([0, T] \times[0, L])^{n}$ to (2.1) satisfies

$$
y(T, x)=z^{1}, \quad \forall x \in[0, L] .
$$

For every $(t, x) \in[0, T] \times[0, L)$, we set

$$
\psi(t) \stackrel{\text { def }}{=} \Theta(t, \bar{X}(t, x)) .
$$

Since $\Theta \in C_{L}^{1}([0, T] \times[0, L])^{m}$, we have $\psi \in C^{1}([0, T])^{m}$, while (2.5) follows from (2.2). Let $z \in C^{1}([0, T])^{n}$ be the solution to (2.4) associated with $\psi$ defined by (2.18). Let $x \in[0, L)$ being fixed. Writing $y$ along the extended characteristic $\bar{X}(\cdot, x)$, we see that $t \in[0, T] \mapsto y(t, \bar{X}(t, x))$ solves (2.4) with $z^{0}=y^{0}(x)$ (at least in the weak sense $\left.W^{1, \infty}(0, T)^{n}\right)$. By uniqueness of the solution to (2.4) we then have

$$
z(t)=y(t, \bar{X}(t, x)), \quad \forall t \in[0, T] .
$$

In particular, $z(T)=z^{1}$ thanks to (2.17). $\mathrm{Q}$

2.2. Semilinear systems. Let us consider the following semilinear first order hyperbolic system with periodic boundary conditions:

$$
\left\{\begin{array}{l}
y_{t}+y_{x}=f(y, \Theta), \quad(t, x) \in[0, T] \times[0, L], \\
y(t, L)=y(t, 0), \quad t \in[0, T] \\
y(0, x)=y^{0}(x), \quad x \in[0, L]
\end{array}\right.
$$

We assume that the nonlinearity $f: \mathbb{R}^{n} \times \mathbb{R}^{m} \longrightarrow \mathbb{R}^{n}$ is of class $C^{2}$.

Definition 2.7. We say that $(\tilde{y}, \tilde{\Theta}) \in C_{L}^{1}([0, T] \times[0, L])^{n} \times C_{L}^{1}([0, T] \times[0, L])^{m}$ is a trajectory of System (2.19) if it is a classical solution to (2.19) for some $y^{0} \in$ $C_{L}^{1}([0, L])^{n}$ and $\Theta$ satisfies (2.2).

Definition 2.8. Let $(\tilde{y}, \tilde{\Theta})$ be trajectory of System (2.19). We say that System (2.19) is locally exactly controllable around the trajectory $(\tilde{y}, \tilde{\Theta})$ at time $T>0$ if, for every $\varepsilon>0$, there exists $\mu>0$ such that, for every $y^{0}, y^{1} \in C_{L}^{1}([0, T] \times[0, L])^{n}$ with

$$
\left\|y^{0}-\tilde{y}(0, \cdot)\right\|_{C^{1}} \leq \mu, \quad\left\|y^{1}-\tilde{y}(T, \cdot)\right\|_{C^{1}} \leq \mu
$$


there exists a control $\Theta \in C_{L}^{1}([0, T] \times[0, L])^{m}$ that satisfies (2.2) and a classical solution $y \in C_{L}^{1}([0, T] \times[0, L])^{n}$ to (2.19) such that

$$
\begin{gathered}
y(T, x)=y^{1}(x), \quad \forall x \in[0, L], \\
\|y-\tilde{y}\|_{C^{1}} \leq \varepsilon \\
\|\Theta-\tilde{\Theta}\|_{C^{1}} \leq \varepsilon .
\end{gathered}
$$

Then, we have the following result. The proof is classical and use the Banach fixed point theorem (see for instance [Cor07, Section 4.1]).

Theorem 2.9. Let $(\tilde{y}, \tilde{\Theta})$ be a trajectory of System (2.19). Assume that the linearization of system (2.19) around the trajectory $(\tilde{y}, \tilde{\Theta})$, that is the linear system

$$
\left\{\begin{array}{l}
y_{t}+y_{x}=\frac{\partial f}{\partial y}(\tilde{y}(t, x), \tilde{\Theta}(t, x)) y+\frac{\partial f}{\partial \Theta}(\tilde{y}(t, x), \tilde{\Theta}(t, x)) \Theta,(t, x) \in[0, T] \times[0, L], \\
y(t, L)=y(t, 0), \quad t \in[0, T], \\
y(0, x)=y^{0}(x), \quad x \in[0, L],
\end{array}\right.
$$

is exactly controllable at time $T>0$. Then, System (2.19) is locally exactly controllable around the trajectory $(\tilde{y}, \tilde{\Theta})$ at time $T$.

3. Quasilinear systems with different velocities. In what follows, we denote by $e_{1}=(1,0), e_{2}=(0,1)$, the canonical basis of $\mathbb{R}^{2}$.

We are now interested in the controllability of the following $2 \times 2$ quasilinear system by one control force

$$
\left\{\begin{array}{l}
y_{t}+\Lambda(y) y_{x}+f(y)=e_{1} \Theta, \quad(t, x) \in[0, T] \times[0, L] \\
y(t, L)=y(t, 0), \quad t \in[0, T] \\
y(0, x)=y^{0}(x), \quad x \in[0, L]
\end{array}\right.
$$

where

$$
\Lambda(y)=\operatorname{diag}\left(\lambda_{1}(y), \lambda_{2}(y)\right), \quad \forall y \in \mathbb{R}^{2},
$$

with

$$
\lambda_{1}(y) \neq 0, \quad \lambda_{2}(y) \neq 0, \quad \forall y \in \mathbb{R}^{2},
$$

and

$$
f(y)=\left(\begin{array}{l}
f_{1}(y) \\
f_{2}(y)
\end{array}\right), \quad \forall y \in \mathbb{R}^{2},
$$

with

$$
f_{1}(0)=f_{2}(0)=0
$$

so that $(0,0)$ is a trajectory of system (3.1). We assume that $\lambda_{1}, \lambda_{2}, f_{1}, f_{2} \in C^{\infty}\left(\mathbb{R}^{2}\right)$. In particular, System (3.1) is hyperbolic (see for instance [LY85, Pages 1-2]). 
Then, for every $T>0$, there exist $C>0$ and $\mu>0$ such that, for every $\Theta \in$ $C_{L}^{k}([0, T] \times[0, L])$ and every $y^{0} \in C_{L}^{k}([0, T] \times[0, L])^{2}\left(k \in \mathbb{N}^{*}\right)$ such that

$$
\|\Theta\|_{C^{k}} \leq \mu, \quad\left\|y^{0}\right\|_{C^{k}} \leq \mu,
$$

there exists a unique semi-global classical solution $y \in C_{L}^{k}([0, T] \times[0, L])^{2}$ to (3.1), and this solution satisfies the estimate

$$
\|y\|_{C^{k}} \leq C\left(\left\|y^{0}\right\|_{C^{k}}+\|\Theta\|_{C^{k}}\right) .
$$

We refer to [LJ01, Wan06] for a proof of this well-posedness result.

The technical point in the method we will develop lies in the algebraic solvability. Since the eigenvalues of $\Lambda(y)$ might be distinct, the more the number $n$ of equations of the system is large, the more it becomes difficult to solve algebraically the system. That is why we restrict ourselves to the case of $n=2$ equations. We also see during this step that we have to take the derivatives of the coefficients of the equations, which shows the loss of derivatives. When $n>2$, the algebraic solvability becomes a difficult task that involves the same arguments as in CL14 to be solved. This is not the purpose of the present paper but this could be the investigation of further developments. On the other hand, once the algebraic solvability is established (under some conditions), the rest of the proof of Theorem 3.1 below remains unchanged whether $n=2,3, \ldots$

Our main result is the following local exact controllability result around the trajectory $(0,0)$.

Theorem 3.1. Assume that

$$
T>(L-(b-a)) \max \left\{\frac{1}{\left|\lambda_{1}(0)\right|}, \frac{1}{\left|\lambda_{2}(0)\right|}\right\},
$$

and

$$
\frac{\partial f_{2}}{\partial y_{1}}(0) \neq 0
$$

Then, for every $\varepsilon>0$, there exists $\mu>0$, such that, for every $y^{0}, y^{1} \in C_{L}^{6}([0, L])^{2}$ that satisfy

$$
\left\|y^{0}\right\|_{C^{6}} \leq \mu, \quad\left\|y^{1}\right\|_{C^{6}} \leq \mu,
$$

there exists a control $\Theta \in C_{L}^{1}([0, T] \times[0, L])$ that satisfy

$$
\begin{gathered}
\operatorname{supp} \Theta \subset[\delta, T-\delta] \times[a+\delta, b-\delta], \\
\|\Theta\|_{C^{1}} \leq \varepsilon,
\end{gathered}
$$

for every $0<\delta<\min (T,(b-a) / 2) / 4$ such that

$$
T-4 \delta>(L-(b-a-8 \delta)) \max \left\{\frac{1}{\left|\lambda_{1}(0)\right|}, \frac{1}{\left|\lambda_{2}(0)\right|}\right\},
$$

and such that the corresponding solution $y \in C_{L}^{1}([0, T] \times[0, L])^{n}$ to (3.1) satisfy

$$
\begin{gathered}
y(T, x)=y^{1}(x), \quad \forall x \in[0, L], \\
\|y\|_{C^{1}} \leq \varepsilon .
\end{gathered}
$$


REMARK 5. The regularity required on the initial and final data (at least $C^{6}$ ) is probably not optimal if we seek a control which is $C^{1}$. Note however that one cannot expect a $C^{1}$-regularity for the control if these data are only $C^{1}$ (where the problem is well-posed though), even in the linear case. Indeed, consider the linear system

$$
\left\{\begin{array}{l}
u_{t}+u_{x}=\Theta, \quad(t, x) \in[0, T] \times[0, L], \\
v_{t}+\lambda v_{x}+u=0, \quad(t, x) \in[0, T] \times[0, L], \\
u(t, L)=u(t, 0), \quad v(t, L)=v(t, 0), \quad t \in[0, T], \\
u(0, x)=u^{0}(x), \quad v(0, x)=v^{0}(x), \quad x \in[0, L],
\end{array}\right.
$$

with $\lambda>1$. Then, for $L-(b-a)<T<L$, writing the system along the characteristics $(t, t+\lambda x)$, we obtain the relation

$$
\begin{aligned}
\int_{0}^{T} \Theta(t, t+\lambda x) d t & =u^{1}(T+\lambda x)-u^{0}(\lambda x) \\
- & (1-\lambda) \lambda\left(\left(v^{1}\right)^{\prime}(T+\lambda x)-\left(v^{0}\right)^{\prime}(\lambda x)\right), \quad \forall x \in[0,(L-T) / \lambda] .
\end{aligned}
$$

This shows that, in order to obtain a $C^{1}$-regularity for $\Theta$, we have to at least increase the regularities of $v^{0}$ and $v^{1}$ to $C^{2}$.

3.1. Controllability by two controls. The starting point of the proof of Theorem 3.1 is to control System (3.1) with 2 internal controls. We are going to use the results of LR03 on the controllability of $n \times n$ quasilinear systems by $n$ boundary controls and an extension method to obtain the following result. Observe the different levels of regularity between the state and the controls.

Proposition 3.2. Let us consider the system

$$
\left\{\begin{array}{l}
y_{t}+\Lambda(y) y_{x}+f(y)=e_{1} \Theta_{1}+e_{2} \Theta_{2}, \quad(t, x) \in[0, T] \times[0, L], \\
y(t, L)=y(t, 0), \quad t \in[0, T] \\
y(0, x)=y^{0}(x), \quad x \in[0, L] .
\end{array}\right.
$$

Assume that (3.2) holds and let (3.5) be satisfied for $\delta / 2$. Then, for every $\varepsilon>0$, there exists $\mu>0$, such that, for every $y^{0}, y^{1} \in C_{L}^{k}([0, L])^{2}(k \geq 2)$ that satisfy

$$
\left\|y^{0}\right\|_{C^{k}} \leq \mu, \quad\left\|y^{1}\right\|_{C^{k}} \leq \mu
$$

there exist controls $\Theta_{1}, \Theta_{2} \in C_{L}^{k-1}([0, T] \times[0, L])$ that satisfy (3.4a) and

$$
\left\|\Theta_{1}\right\|_{C^{k-1}}+\left\|\Theta_{2}\right\|_{C^{k-1}} \leq \varepsilon
$$

and such that the corresponding solution $y \in C_{L}^{k}([0, T] \times[0, L])^{n}$ to (3.7) satisfy

$$
\begin{gathered}
y(T, x)=y^{1}(x), \quad \forall x \in[0, L], \\
\|y\|_{C^{k}} \leq \varepsilon .
\end{gathered}
$$


Proof. We treat only the case where $\lambda_{1}(y)<0<\lambda_{2}(y)$ for every $y \in \mathbb{R}^{2}$, the other cases being similar. Let us then consider the following boundary control problem on the domain $[0, T] \times[b-\delta, a+\delta+L]$ :

$$
\left\{\begin{array}{l}
y_{t}^{*}+\Lambda\left(y^{*}\right) y_{x}^{*}+f\left(y^{*}\right)=0, \quad(t, x) \in[0, T] \times[b-\delta, a+\delta+L], \\
y_{1}^{*}(t, a+\delta+L)=H_{1}(t), \quad y_{2}^{*}(t, b-\delta)=H_{2}(t), \quad t \in[0, T], \\
y^{*}(0, x)=\overline{y^{0}}(x), \quad x \in[b-\delta, a+\delta+L], \\
y^{*}(T, x)=\overline{y^{1}}(x), \quad x \in[b-\delta, a+\delta+L],
\end{array}\right.
$$

where $H_{1}, H_{2} \in C^{k}([0, T])$ are boundary controls, and where we have extended by periodicity $y^{0}$ and $y^{1}$ to

$$
\begin{aligned}
& \overline{y^{0}}(x) \stackrel{\text { def }}{=}\left\{\begin{array}{cc}
y^{0}(x) & \text { if } x \in[b-\delta, L], \\
y^{0}(x-L) & \text { if } x \in[L, a+\delta+L],
\end{array}\right. \\
& \overline{y^{1}}(x) \stackrel{\text { def }}{=}\left\{\begin{array}{cc}
y^{1}(x) & \text { if } x \in[b-\delta, L], \\
y^{1}(x-L) & \text { if } x \in[L, a+\delta+L] .
\end{array}\right.
\end{aligned}
$$

Note that, since $y_{0}, y_{1} \in C_{L}^{k}([0, L])^{n}$, we have $\overline{y^{0}}, \overline{y^{1}} \in C^{k}([b-\delta, a+\delta+L])^{2}$. Since, by assumption, $T$ satisfies

$$
T>(L-(b-a-2 \delta)) \max \left\{\frac{1}{\left|\lambda_{1}(0)\right|}, \frac{1}{\left|\lambda_{2}(0)\right|}\right\},
$$

by [LR03, Theorem 1.2], for every $\mu>0$ small enough, for every $y^{0}, y^{1}$ such that

$$
\left\|y^{0}\right\|_{C^{k}} \leq \mu, \quad\left\|y^{1}\right\|_{C^{k}} \leq \mu,
$$

there exists $y^{*} \in C^{k}([0, T] \times[b-\delta, a+\delta+L])^{2}$ that satisfies (3.9) with $\left\|y^{*}\right\|_{C^{k}}$ small. Let $y^{* *}$ be any $C^{k}([0, T] \times[0, L])^{2}$ function such that $\left\|y^{* *}\right\|_{C^{k}} \leq\left\|y^{*}\right\|_{C^{k}}$ and

$$
y^{* *}(t, x)=\left\{\begin{array}{cc}
y^{*}(t, x+L) & \text { if } x \in[0, a+\delta], \\
y^{*}(t, x) & \text { if } x \in[b-\delta, L] .
\end{array}\right.
$$

On the other hand, let us introduce $\bar{y} \in C^{k}([0, T] \times[0, L])^{2}$ defined by

$$
\bar{y}(t, x) \stackrel{\text { def }}{=} \eta_{1}(t) u(t, x)+\eta_{2}(t) v(t, x)
$$

where $\eta_{1}, \eta_{2} \in C^{\infty}([0, T])$ are time cut-off functions with $0 \leq \eta_{i} \leq 1$ and

$$
\begin{array}{llll}
\eta_{1}(0)=1, & \eta_{1}(T)=0, & \eta_{1}^{(i)}(0)=\eta_{1}^{(i)}(T)=0, & \forall i \in \llbracket 1, k+1 \rrbracket, \\
\eta_{2}(0)=0, & \eta_{2}(T)=1, & \eta_{2}^{(i)}(0)=\eta_{2}^{(i)}(T)=0, & \forall i \in \llbracket 1, k+1 \rrbracket,
\end{array}
$$

and $u, v \in C^{k}([0, T] \times[0, L])^{2}$ are the forward and backward solutions to the free evolving systems

$$
\left\{\begin{array}{l}
u_{t}+\Lambda(u) u_{x}+f(u)=0, \quad(t, x) \in[0, T] \times[0, L], \\
u(t, L)=u(t, 0), \quad t \in[0, T] \\
u(0, x)=y^{0}(x), \quad x \in[0, L]
\end{array}\right.
$$


and

$$
\left\{\begin{array}{l}
v_{t}+\Lambda(v) v_{x}+f(v)=0, \quad(t, x) \in[0, T] \times[0, L], \\
v(t, L)=v(t, 0), \quad t \in[0, T] \\
v(T, x)=y^{1}(x), \quad x \in[0, L]
\end{array}\right.
$$

Let now $\xi \in C^{\infty}([0, L])$ be a space cut-off function with $0 \leq \xi \leq 1$ and

$$
\xi(x)= \begin{cases}1 & \text { if } x \in[0, a] \cup[b, L], \\ 0 & \text { if } x \in[a+\delta, b-\delta] .\end{cases}
$$

Let $y$ and $\Theta$ be defined by

$$
y(t, x) \stackrel{\text { def }}{=} \xi(x) y^{* *}(t, x)+(1-\xi(x)) \bar{y}(t, x)
$$

and

$$
\Theta \stackrel{\text { def }}{=} y_{t}+\Lambda(y) y_{x}+f(y) .
$$

By construction, $y \in C^{k}([0, T] \times[0, L])^{2}$ and $\Theta \in C^{k-1}([0, T] \times[0, L])^{2}$. Still by construction, $(y, \Theta)$ solves (3.7), $y$ satisfies (3.8a) and $\Theta$ satisfies

$$
\begin{gathered}
\operatorname{supp} \Theta \subset[0, T] \times[a, b], \\
\partial_{t}^{i} \Theta(0, \cdot)=\partial_{t}^{i} \Theta(T, \cdot)=0, \quad \forall i \in \llbracket 0, k+1 \rrbracket .
\end{gathered}
$$

The smallnesses of $y$ and $\Theta$ follow from the smallnesses of $y^{*}$ and $\bar{y}$.

To obtain (3.4a) we let system (3.7) evolves freely (without control), forward on the domain $[0, \delta] \times[0, L]$ and backward on the domain $[T-\delta, T] \times[0, L]$, and denote by $y^{\delta}(x)\left(\right.$ resp. $\left.y^{T-\delta}(x)\right)$ its value at time $t=\delta($ resp. $t=T-\delta)$. By the previous step, replacing $[a, b]$ by $[a+\delta, b-\delta]$, there exists a control $\Theta$ with (3.4a) and

$$
\partial_{t}^{i} \Theta(\delta, \cdot)=\partial_{t}^{i} \Theta(T-\delta, \cdot)=0, \quad \forall i \in \llbracket 0, k+1 \rrbracket,
$$

that steers the solution to System (3.7), posed on the time reduced domain $[\delta, T-\delta] \times$ $[0, L]$, from $y^{\delta}$ to $y^{T-\delta}$. Thus, we can extend $\Theta$ by zero outside $(\delta, T-\delta) \times(a+\delta, b-\delta)$. Note that the smallnesses are preserved. $\square$

3.2. Algebraic solvability. In this section we recall the notion of algebraic solvability and the fixed point theorem of M. Gromov. We refer to Gro86, Section $2.3]$ for more details.

In what follows $Q$ is a smooth bounded open subset of $\mathbb{R}^{2}$ and $\mathscr{D}: C^{r}(\bar{Q})^{p} \longrightarrow$ $C^{0}(\bar{Q})^{q}\left(p, q \in \mathbb{N}^{*}\right)$ is a nonlinear $C^{\infty}$-differential operator of order $r \in \mathbb{N}^{*}$. We recall that this means that there exists a $C^{\infty}$-function $F: \mathbb{R}^{n_{r, p}} \longrightarrow \mathbb{R}^{q}$, where $n_{r, p}=2+p$ card $\left\{\left(\alpha_{1}, \alpha_{2}\right) \in \mathbb{N}^{2} \mid \alpha_{1}+\alpha_{2} \leq r\right\}$, such that $\mathscr{D}$ writes

$$
\mathscr{D}(z)=F\left(J^{r} z\right), \quad \forall z \in C^{r}(\bar{Q})^{p},
$$

where $J^{r} z$ denotes the $r$-jet of $z$, that is the function defined for every $(t, x) \in \bar{Q}$ by

$$
J^{r} z(t, x) \stackrel{\text { def }}{=}\left((t, x), z(t, x), \ldots, \frac{\partial^{|\alpha|} z}{\partial t^{\alpha_{1}} \partial x^{\alpha_{2}}}(t, x), \ldots, \frac{\partial^{r} z}{\partial t^{\alpha_{1}} \partial x^{\alpha_{2}}}(t, x)\right) \in \mathbb{R}^{n_{r, p}} .
$$


Clearly, the map $\mathscr{D}$ is of class $C^{\infty}$ and we denote by $\mathscr{L}_{z}: C^{r}(\bar{Q})^{p} \longrightarrow C^{0}(\bar{Q})^{q}$ its differential at $z \in C^{r}(\bar{Q})^{p}$.

Definition 3.3. We say that $\mathcal{A}$ is a differential relation of order $d(d \in \mathbb{N})$ if there exists $\mathcal{R} \subset \mathbb{R}^{n_{d, p}}$ such that

$$
\mathcal{A} \stackrel{\text { def }}{=}\left\{z \in C^{d}(\bar{Q})^{p} \mid J^{d} z \in \mathcal{R}, \quad \forall(t, x) \in \bar{Q}\right\} .
$$

It is said to be open if it is an open subset of $C^{d}(\bar{Q})^{p}$.

Definition 3.4. Let $\mathcal{A} \subset C^{d}(\bar{Q})^{p}$ be a differential relation of order d. We say that the operator $\mathscr{D}$ admits an infinitesimal inversion of order $s \in \mathbb{N}$ over $\mathcal{A}$ if there exists a family of linear differential operators of order $s, \mathscr{M}_{z}: C^{s}(\bar{Q})^{q} \longrightarrow C^{0}(\bar{Q})^{p}$, $z \in \mathcal{A}$, such that:

1. For every $g \in C^{s}(\bar{Q})^{q}$ being fixed, $z \mapsto \mathscr{M}_{z}(g)$ is a differential operator of order d (possibly nonlinear) and it is a $C^{\infty}$-differential operator in $(z, g)$.

2. Algebraic solvability: for every $z \in \mathcal{A}^{d+s} \stackrel{\text { def }}{=} \mathcal{A} \cap C^{d+s}(\bar{Q})^{p}$, we have

$$
\mathscr{L}_{z} \circ \mathscr{M}_{z}=\operatorname{Id}_{C^{r+s}(\bar{Q})^{q}},
$$

The proof of Theorem 3.1 is based on the following result Gro86, Section 2.3.2, Main Theorem]:

THEOREM 3.5. Let $\mathcal{A} \subset C^{d}(\bar{Q})^{p}$ be a nonempty open differential relation of order d. Assume that $\mathscr{D}$ admits an infinitesimal inversion of order $s$ over $\mathcal{A}$. Let

$$
\begin{gathered}
\sigma_{0}>\max (d, 2 r+s) \\
\nu \in(0,+\infty)
\end{gathered}
$$

Then, there exist a family of sets $\mathcal{B}_{z} \subset C^{\sigma_{0}+s}(\bar{Q})^{q}$ and a family of operators $\mathscr{D}_{z}^{-1}$ : $\mathcal{B}_{z} \longrightarrow \mathcal{A}$, where $z \in \mathcal{A}^{\sigma_{0}+r+s}$, such that the following properties hold.

1. Neighborhood property: for every $z \in \mathcal{A}^{\sigma_{0}+r+s}$, we have $0 \in \mathcal{B}_{z}$ and the set $\mathcal{B}$ defined by

$$
\mathcal{B}=\bigcup_{z \in \mathcal{A}^{\sigma_{0}+r+s}}\{z\} \times \mathcal{B}_{z},
$$

is an open subset of $C^{\sigma_{0}+r+s}(\bar{Q})^{p} \times C^{\sigma_{0}+s}(\bar{Q})^{q}$.

2. Inversion property:

$$
\mathscr{D}\left(\mathscr{D}_{z}^{-1}(g)\right)=\mathscr{D}(z)+g, \quad \forall(z, g) \in \mathcal{B} .
$$

3. Normalization property:

$$
\mathscr{D}_{z}^{-1}(0)=z, \quad \forall z \in \mathcal{A}^{\sigma_{0}+r+s} .
$$

4. Locality: for every $(t, x) \in \bar{Q}$ and for every $\left(z_{1}, g_{1}\right),\left(z_{2}, g_{2}\right) \in \mathcal{B}$, if we have

$$
\left(z_{1}, g_{1}\right)(\tilde{t}, \tilde{x})=\left(z_{2}, g_{2}\right)(\tilde{t}, \tilde{x}), \quad \forall(\tilde{t}, \tilde{x}) \in \mathrm{B}((t, x), \nu) \cap \bar{Q},
$$

then,

$$
\mathscr{D}_{z_{1}}^{-1}\left(g_{1}\right)(t, x)=\mathscr{D}_{z_{2}}^{-1}\left(g_{2}\right)(t, x)
$$


3.3. Proof of Theorem 3.1. All along this section, $\delta>0$ is a fixed real number such that (3.5) holds. Let us denote

$$
Q_{\delta} \stackrel{\text { def }}{=}(\delta, T-\delta) \times(a+\delta, b-\delta),
$$

and let $Q$ be an open set of $\mathbb{R} \times \mathbb{R}$ of class $C^{\infty}$ such that (see Fig. 3.1 below)

$$
\overline{Q_{\delta}} \subset Q, \quad \bar{Q} \subset(0, T) \times(a, b) .
$$

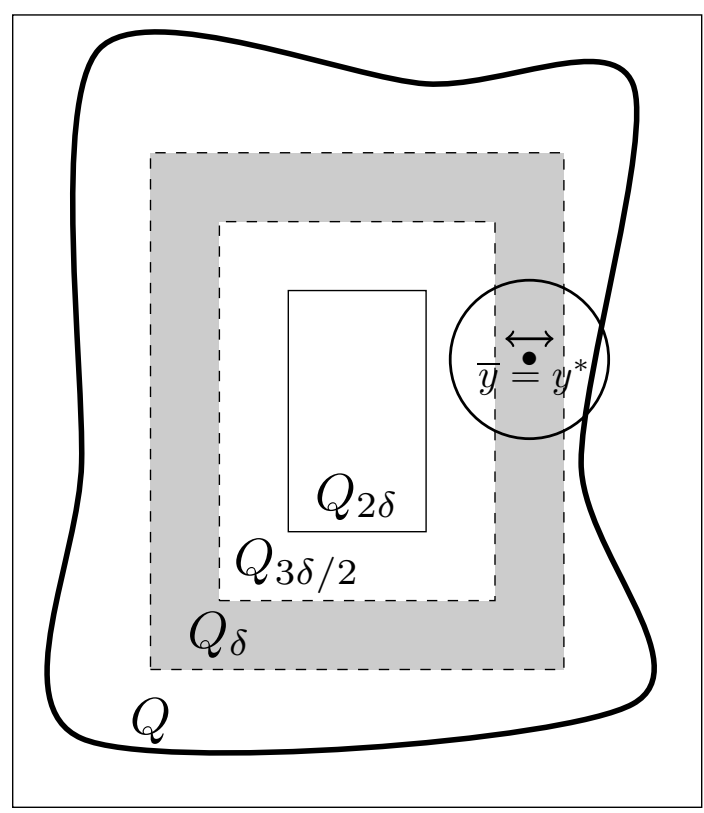

Fig. 3.1: Matching $\bar{y}$ to $y^{*}$.

Let us introduce the operator $\mathscr{D}$ defined by

$$
\begin{aligned}
\mathscr{D}: C^{1}(\bar{Q})^{3} & \longrightarrow C^{0}(\bar{Q})^{2} \\
(y, \Theta) & \longmapsto y_{t}+\Lambda(y) y_{x}+f(y)-e_{1} \Theta .
\end{aligned}
$$

This is a nonlinear $C^{\infty}$-differential operator of order 1 . Note that $(y, \Theta)$ solves the equation of (3.1) if, and only if,

$$
\mathscr{D}(y, \Theta)=0
$$

Let $\mathscr{L}_{(\tilde{y}, \tilde{\Theta})}$ be the differential of the operator $\mathscr{D}$ at $(\tilde{y}, \tilde{\Theta}) \in C^{1}(\bar{Q})^{3}$ :

$$
\begin{aligned}
\mathscr{L}_{(\tilde{y}, \tilde{\Theta})}: C^{1}(\bar{Q})^{3} & \longrightarrow C^{0}(\bar{Q})^{2} \\
(y, \Theta) & \longmapsto y_{t}+\Lambda(\tilde{y}) y_{x}+\left(\Lambda^{\prime}(\tilde{y}) y\right) \tilde{y}_{x}+f^{\prime}(\tilde{y}) y-e_{1} \Theta .
\end{aligned}
$$


This is a nonlinear $C^{\infty}$-differential operator of order 1 in $(\tilde{y}, \tilde{\Theta})$.

Let us denote

$$
\begin{gathered}
y=\left(\begin{array}{l}
u \\
v
\end{array}\right), \quad \tilde{y}=\left(\begin{array}{l}
\tilde{u} \\
\tilde{v}
\end{array}\right), \\
\left(\Lambda^{\prime}(\tilde{y}) y\right) \tilde{y}_{x}+f^{\prime}(\tilde{y}) y=\left(\begin{array}{ll}
a_{11} & a_{12} \\
a_{21} & a_{22}
\end{array}\right)\left(\begin{array}{l}
u \\
v
\end{array}\right) .
\end{gathered}
$$

We have

$$
a_{11}=\frac{\partial \lambda_{1}}{\partial u}(\tilde{y}) \frac{\partial \tilde{u}}{\partial x}+\frac{\partial f_{1}}{\partial u}(\tilde{y}), \quad a_{21}=\frac{\partial \lambda_{2}}{\partial u}(\tilde{y}) \frac{\partial \tilde{u}}{\partial x}+\frac{\partial f_{2}}{\partial u}(\tilde{y}) .
$$

Proposition 3.6. Let $\mathcal{A}$ be defined by

$$
\mathcal{A} \stackrel{\text { def }}{=}\left\{(\tilde{y}, \tilde{\Theta}) \in C^{2}(\bar{Q})^{3} \mid a_{21}(t, x) \neq 0, \quad \forall(t, x) \in \bar{Q}\right\},
$$

(clearly, $\mathcal{A}$ is an open differential relation of order 2). Then, the operator $\mathscr{D}$ admits an infinitesimal inversion of order 1 over $\mathcal{A}$.

Proof. Let $(\tilde{y}, \tilde{\Theta}) \in \mathcal{A}$. Let $\left(g_{1}, g_{2}\right) \in C^{1}(\bar{Q})^{2}$. We have to solve the equation $\mathscr{L}_{(\tilde{y}, \tilde{\Theta})}(y, \Theta)=\left(g_{1}, g_{2}\right)$ in such a way that $(y, \Theta)$ is a linear combination of derivatives of $g_{1}$ and $g_{2}$. The equation $\mathscr{L}_{(\tilde{y}, \tilde{\Theta})}(y, \Theta)=\left(g_{1}, g_{2}\right)$ rewrites as

$$
\left\{\begin{array}{lll}
u_{t}+\lambda_{1}(\tilde{y}) u_{x}+a_{11} u+a_{12} v-\Theta & =g_{1}, & \text { in } \bar{Q} \\
v_{t}+\lambda_{2}(\tilde{y}) v_{x}+a_{21} u+a_{22} v & =g_{2}, & \text { in } \bar{Q} .
\end{array}\right.
$$

By definition of $\mathcal{A}$, we have

$$
a_{21}(t, x) \neq 0, \quad \forall(t, x) \in \bar{Q}
$$

In this case, the algebraic solvability is not very difficult: we first put

$$
v=0,
$$

so that the second equation simply becomes $a_{21} u=g_{2}$. As a result, using (3.20) we can take

$$
u=\frac{1}{a_{21}} g_{2} .
$$

Finally, it remains to set

$$
\Theta=-g_{1}+\frac{1}{a_{21}}\left(\left(g_{2}\right)_{t}+\lambda_{1}(\tilde{y})\left(g_{2}\right)_{x}\right)+\left(\left(\frac{1}{a_{21}}\right)_{t}+\lambda_{1}(\tilde{y})\left(\frac{1}{a_{21}}\right)_{x}+\frac{a_{11}}{a_{21}}\right) g_{2} .
$$

(Note that (3.23), together with (3.18), shows why $C^{2}(\bar{Q})^{3}$ cannot be replaced by $C^{1}(\bar{Q})^{3}$ in Proposition 3.6.) 
Then, the following family of differential operators satisfies all the required properties $(s=1)$ to be an infinitesimal inversion of $\mathscr{D}$ over $\mathcal{A}$ :

$$
\begin{aligned}
\mathscr{M}_{(\tilde{y}, \tilde{\Theta})}: C^{1}(\bar{Q})^{2} & \longrightarrow C^{0}(\bar{Q})^{3} \\
\left(g_{1}, g_{2}\right) & \longmapsto((u, v), \Theta),
\end{aligned}
$$

where $u, v$ and $\Theta$ are respectively defined by (3.22), (3.21) and (3.23).

Proof of Theorem 3.1. Let $\varepsilon>0$ be fixed. Let $\mathcal{A}$ be the open differential relation of order 2 defined by (3.19) and set

$$
\mathcal{A}_{\varepsilon} \stackrel{\text { def }}{=} \mathcal{A} \cap\left\{(\tilde{y}, \tilde{\Theta}) \in C^{1}(\bar{Q})^{3} \quad \mid\|\tilde{y}\|_{C^{1}}<\varepsilon,\|\tilde{\Theta}\|_{C^{1}}<\varepsilon\right\} .
$$

Note that $\mathcal{A}_{\varepsilon}$ is an open differential relation of order 2 which is nonempty since $0 \in \mathcal{A}_{\varepsilon}$ by assumption (3.3). We choose

$$
\begin{gathered}
\nu \stackrel{\text { def }}{=} \frac{\delta}{2}, \\
d=2, r=1, s=1, \sigma_{0}=4 .
\end{gathered}
$$

Note that, from (3.25), one gets (3.14) and, from (3.26), one gets (3.13).

Thanks to Proposition 3.6 $\mathscr{D}$ admits an infinitesimal inversion of order 1 over $\mathcal{A}_{\varepsilon}$. Thus, we can apply Theorem 3.5 which provides a family of sets $\mathcal{B}_{(\tilde{y}, \tilde{\Theta})} \subset C^{5}(\bar{Q})^{2}$ and a family of operators $\mathscr{D}_{(\tilde{y}, \tilde{\Theta})}^{-1}: \mathcal{B}_{(\tilde{y}, \tilde{\Theta})} \longrightarrow \mathcal{A}_{\varepsilon}$, where $(\tilde{y}, \tilde{\Theta}) \in \mathcal{A}_{\varepsilon}^{6} \stackrel{\text { def }}{=} \mathcal{A}_{\varepsilon} \cap C^{6}(\bar{Q})^{3}$, such that all the properties listed in this theorem hold.

Since $0 \in \mathcal{A}_{\varepsilon}, \mathcal{B}$ also contains 0 . Since $\mathcal{B}$ is open, there exists $\rho>0$ such that

$$
\left\{((\tilde{y}, \tilde{\Theta}), g) \in C^{6}(\bar{Q})^{3} \times C^{5}(\bar{Q})^{2} \quad \mid\|\tilde{y}\|_{C^{6}} \leq \rho,\|\tilde{\Theta}\|_{C^{6}} \leq \rho,\|g\|_{C^{5}} \leq \rho\right\} \subset \mathcal{B} .
$$

On the other hand, by Proposition 3.2, there exists $\mu>0$ such that, for every $y^{0}, y^{1} \in$ $C_{L}^{6}([0, L])^{2}$ that satisfy

$$
\left\|y^{0}\right\|_{C^{6}} \leq \mu, \quad\left\|y^{1}\right\|_{C^{6}} \leq \mu,
$$

there exist $\Theta_{1}^{*}, \Theta_{2}^{*} \in C^{5}([0, T] \times[0, L])$ and $y^{*} \in C^{6}([0, T] \times[0, L])^{2}$ such that

$$
\begin{gathered}
\mathscr{D}\left(y^{*}, 0\right)=-e_{1} \Theta_{1}^{*}-e_{2} \Theta_{2}^{*}, \\
y^{*}(T, x)=y^{1}(x), \quad \forall x \in[0, L], \\
\operatorname{supp} \Theta_{1}^{*} \subset Q_{2 \delta}, \quad \operatorname{supp} \Theta_{2}^{*} \subset Q_{2 \delta},
\end{gathered}
$$

and

$$
\left\|y^{*}\right\|_{C^{6}}<\varepsilon^{\prime}, \quad\left\|\Theta_{1}^{*}\right\|_{C^{5}}+\left\|\Theta_{2}^{*}\right\|_{C^{5}}<\varepsilon^{\prime},
$$

where $\varepsilon^{\prime}=\min (\varepsilon, \rho)$. In particular,

$$
\left(y^{*}, 0,-e_{1} \Theta_{1}^{*}-e_{2} \Theta_{2}^{*}\right) \in \mathcal{B},
$$


and we can set

$$
(\bar{y}, \bar{\Theta}) \stackrel{\text { def }}{=} \mathscr{D}_{\left(y^{*}, 0\right)}^{-1}\left(-e_{1} \Theta_{1}^{*}-e_{2} \Theta_{2}^{*}\right) .
$$

By definition $(\bar{y}, \bar{\Theta}) \in C^{1}(\bar{Q})^{3}$ and satisfies

$$
\mathscr{D}(\bar{y}, \bar{\Theta})=\mathscr{D}\left(y^{*}, 0\right)-e_{1} \Theta_{1}^{*}-e_{2} \Theta_{2}^{*}=0 .
$$

Let us now prove that we can match $\bar{y}$ to $y^{*}$ and $\bar{\Theta}=0$ in the open neighborhood $Q_{\delta} \backslash \bar{Q}_{3 \delta / 2}$ (see Fig. 3.1 below). Let then $(t, x) \in Q_{\delta} \backslash \bar{Q}_{3 \delta / 2}$ be fixed and let us show that

$$
\bar{y}(t, x)=y^{*}(t, x), \quad \bar{\Theta}(t, x)=0 .
$$

Since $\Theta_{1}^{*}$ and $\Theta_{2}^{*}$ are supported in $\bar{Q}_{2 \delta}$, we have

$$
\left(y^{*}, 0,-e_{1} \Theta_{1}^{*}-e_{2} \Theta_{2}^{*}\right)(\tilde{t}, \tilde{x})=\left(y^{*}, 0,0\right)(\tilde{t}, \tilde{x}), \quad \forall(\tilde{t}, \tilde{x}) \in \mathrm{B}((t, x), \delta / 2),
$$

and $\left(y^{*}, 0,-e_{1} \Theta_{1}^{*}-e_{2} \Theta_{2}^{*}\right),\left(y^{*}, 0,0\right) \in \mathcal{B}$. Thus, by (3.25), the locality and normalization properties, we obtain (3.28). To conclude the proof, it remains to set

$$
y(t, x) \stackrel{\text { def }}{=}\left\{\begin{array}{cl}
\bar{y}(t, x) & \text { if }(t, x) \in Q_{\delta}, \\
y^{*}(t, x) & \text { if }(t, x) \in[0, T] \times[0, L] \backslash Q_{\delta},
\end{array}\right.
$$

and

$$
\Theta(t, x) \stackrel{\text { def }}{=}\left\{\begin{array}{cl}
\bar{\Theta}(t, x) & \text { if }(t, x) \in Q_{\delta}, \\
0 & \text { if }(t, x) \in[0, T] \times[0, L] \backslash Q_{\delta} .
\end{array}\right.
$$

Then, $(y, \Theta)$ solves (3.1). Since $y=y^{*}$ near the boundary of $[0, T] \times[0, L]$ it satisfies the same periodic boundary conditions $y(\cdot, L)=y(\cdot, 0)$, the same initial condition $y(0, \cdot)=$ $y^{0}$ and the same final condition $y(T, \cdot)=y^{1}$. Finally, note that the smallnesses of $y$ and $\Theta$ follow from the smallness of $y^{*}$ and the definition (3.24) of $\mathcal{A}_{\varepsilon}$.

\section{Appendix. Proof of Lemma 2.6.}

Let us compute $\tau_{k}(x, b)-\tau_{k}(x, a)$ for every $k \in \llbracket k_{\min }(x, b), k_{\max }(x, a) \rrbracket$ and for every $x \in[0, L)$ :

$$
\begin{aligned}
& \tau_{k}(x, b)-\tau_{k}(x, a)= \\
& \left\{\begin{array}{cll}
b-x+k L & \text { if } a-x+k L \in(-\infty, 0) & \text { and } b-x+k L \in[0, T], \\
T & \text { if } a-x+k L \in(-\infty, 0) & \text { and } b-x+k L \in(T,+\infty), \\
b-a & \text { if } a-x+k L \in[0, T] & \text { and } b-x+k L \in[0, T], \\
T-a+x-k L & \text { if } a-x+k L \in[0, T] & \text { and } b-x+k L \in(T,+\infty) .
\end{array}\right.
\end{aligned}
$$

Thus, we see that the main problem that we have to handle is the fact that the length $\tau_{0}(x, b)-\tau_{0}(x, a)\left(\right.$ resp. $\left.\quad \tau_{\left\lceil\frac{T-a}{L}\right\rceil}(x, b)-\tau_{\left\lceil\frac{T-a}{L}\right\rceil}(x, a)\right)$ goes to zero as $x$ goes to $b^{-}$ (resp. $\left.p(a)^{+}\right)$. We are going to approximate uniformly the Gramian associated with (2.4) -(2.5) by modifying it a little bit near the points $b$ and $p(a)$ in order to avoid the 
aforementioned problems. This has to be done in such a way that the invertibility is preserved. Let

$$
\ell_{0}=\min \left(T-a, b-a, L-p(a), b-L+\left\lceil\frac{T-a}{L}\right\rceil L\right) / 2,
$$

(note that $\ell_{0}$ is positive thanks to $\left(\mathcal{H}_{1}\right)$. Then, for every $x \in[0, L)$ and $0 \leq \ell<\ell_{0}$ we introduce the approximated Gramian

$$
Q(x, \ell)=Q_{0}(x, \ell)+\sum_{k=1}^{\left\lceil\frac{T-a}{L}\right\rceil-1} Q_{k}(x, \ell)+Q_{\left\lceil\frac{T-a}{L}\right\rceil}(x, \ell),
$$

where, for every $k \in \llbracket 1,\left\lceil\frac{T-a}{L}\right\rceil-1 \rrbracket$,

$$
Q_{k}(x, \ell)=\int_{\tau_{k}(x, a)+\ell}^{\tau_{k}(x, b)-\ell} G(s, x) d s,
$$

with $G(s, x)=R_{x}(T, s) B_{x}(s) B_{x}(s)^{*} R_{x}(T, s)^{*}$, and

$$
Q_{0}(x, \ell)=\left\{\begin{array}{cl}
\int_{\tau_{0}(x, a)+\ell}^{\tau_{0}(x, b)-\ell} G(s, x) d s & \text { if } x \in[0, b-2 \ell), \\
0 & \text { if } x \in[b-2 \ell, L),
\end{array}\right.
$$

and where

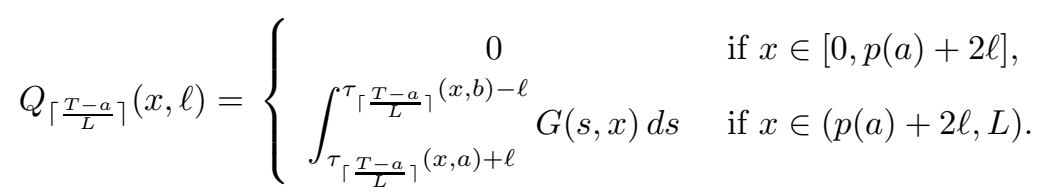

Step 1: Let us prove in a first time that there exists $r>0$ such that, for every $x \in[0, L)$,

$$
\mathrm{B}(Q(x, 0), r) \subset \mathrm{GL}_{n}(\mathbb{R}) .
$$

where $\mathrm{GL}_{n}(\mathbb{R})$ denotes again the set of invertible matrices of size $n$ and $\mathrm{B}(M, \rho)$ the open ball of center $M \in \mathbb{R}^{n \times n}$ and radius $\rho>0$. Since $\lim _{\substack{x \rightarrow L \\ x<L}} Q(x, 0)=Q(0,0)$, we can extend $Q(\cdot, 0)$ by continuity to $[0, L]$ (still denoted by $Q(\cdot, 0))$ with $Q(L, 0)=$ $Q(0,0)$. By assumption $\left(\mathcal{H}_{2}\right)$, for every $x \in[0, L], Q(x, 0) \in \mathrm{GL}_{n}(\mathbb{R})$. Since $\mathrm{GL}_{n}(\mathbb{R})$ is open, there exists $r(x)>0$ such that

$$
\mathrm{B}(Q(x, 0), r(x)) \subset \mathrm{GL}_{n}(\mathbb{R}) .
$$

On the other hand,

$$
Q([0, L], 0) \subset \bigcup_{x \in[0, L]} \mathrm{B}\left(Q(x, 0), \frac{r(x)}{2}\right),
$$

and $Q([0, L], 0)$ is compact (by continuity of $Q(\cdot, 0)$ on all $[0, L])$, so that there exists $x_{1}, \ldots, x_{q} \in[0, L]$ such that

$$
Q([0, L], 0) \subset \bigcup_{x \in\left\{x_{1}, \ldots, x_{q}\right\}} \mathrm{B}\left(Q(x, 0), \frac{r(x)}{2}\right) .
$$


We define

$$
r=\min \left\{\frac{r\left(x_{1}\right)}{2}, \ldots, \frac{r\left(x_{q}\right)}{2}\right\} .
$$

Thus, for every $x \in[0, L]$, for every $M \in \mathrm{B}(Q(x, 0), r)$, there exists $x_{i} \in\left\{x_{1}, \ldots, x_{q}\right\}$, such that

$$
\left\|M-Q\left(x_{i}, 0\right)\right\| \leq\|M-Q(x, 0)\|+\left\|Q(x, 0)-Q\left(x_{i}, 0\right)\right\|<r+\frac{r\left(x_{i}\right)}{2} \leq r\left(x_{i}\right),
$$

that is $M \in \mathrm{B}\left(Q\left(x_{i}, 0\right), r\left(x_{i}\right)\right)$ and shows that $M$ is invertible by (A.2).

Step 2: By construction we have

$$
\sup _{x \in[0, L)}\|Q(x, \ell)-Q(x, 0)\| \underset{\ell \rightarrow 0}{\longrightarrow} 0 .
$$

Thus, there exists $\delta>0$ small enough such that, for every $x \in[0, L)$, we have

$$
Q(x, 2 \delta) \in \mathrm{B}(Q(x, 0), r),
$$

which shows that $Q(x, 2 \delta)$ is invertible by (A.1).

Step 3: Let us now define the cut-off function $\eta$ (see Fig. 2.3). Let us introduce

$$
\begin{aligned}
& \mathcal{T}_{0}(\delta) \stackrel{\text { def }}{=} \\
& \left\{(t, x) \in(0, T) \times(0, L) \quad \mid \quad x \in(0, b-2 \delta), \quad t \in\left(\tau_{0}(x, a)+\delta, \tau_{0}(x, b)-\delta\right)\right\}, \\
& \mathcal{T}_{\left\lceil\frac{T-a}{L}\right\rceil}(\delta) \stackrel{\text { def }}{=}\{(t, x) \in(0, T) \times(0, L), \quad \mid \\
& \left.x \in(p(a)+2 \delta, L), \quad t \in\left(\tau_{\left\lceil\frac{T-a}{L}\right\rceil}(x, a)+\delta, \tau_{\left\lceil\frac{T-a}{L}\right\rceil}(x, b)-\delta\right)\right\},
\end{aligned}
$$

and, for $k \in \llbracket 1,\left\lceil\frac{T-a}{L}\right\rceil-1 \rrbracket$,

$$
\mathcal{T}_{k}(\delta) \stackrel{\text { def }}{=}\left\{(t, x) \in(0, T) \times(0, L) \quad \mid \quad t \in\left(\tau_{k}(x, a)+\delta, \tau_{k}(x, b)-\delta\right)\right\} .
$$

Let $\xi \in C^{1}([0, T] \times[0, L])$ be a cut-off function with $0 \leq \xi \leq 1$ and such that (see Fig. 2.2 to help)

$$
\begin{gathered}
\xi \equiv 1 \text { in } \bigcup_{k=0}^{\left\lceil\frac{T-a}{L}\right\rceil} \mathcal{T}_{k}(2 \delta), \\
\xi \equiv 1 \text { in } \bigcup_{k=0}^{\left\lceil\frac{T-a}{L}\right\rceil-1}\left\{(t, 0) \mid t \in\left(\tau_{k}(0, a)+2 \delta, \tau_{k}(0, b)-2 \delta\right)\right\}, \\
\xi \equiv 1 \text { in } \bigcup_{k=1}^{\left\lceil\frac{T-a}{L}\right\rceil}\left\{(t, L) \mid t \in\left(\tau_{k-1}(0, a)+2 \delta, \tau_{k-1}(0, b)-2 \delta\right)\right\}, \\
\xi \equiv 0 \text { in }[0, T] \times[0, L] \backslash\left(\bigcup_{k=0}^{\left\lceil\frac{T-a}{L}\right\rceil} \overline{\mathcal{T}_{k}(\delta)}\right) .
\end{gathered}
$$


For every $x \in[0, L]$, let us define

$$
Q_{x}=\int_{0}^{T} G(s, x) \xi(s, x) d s .
$$

Note that $Q_{L}=Q_{0}$. Let us show that $Q_{x}$ is invertible for every $x \in[0, L]$. First, note that the controllability Gramian $Q_{x}$ is a nonnegative symmetric matrix. Thus, it is invertible if, and only, if it is positive definite. Let $v \in \mathbb{R}^{n}$. Using $\xi \geq 0$ and (A.3a)-A.3d, we have

$$
\begin{aligned}
v \cdot Q_{x} v & =\int_{0}^{T}\left\|B(s)^{*} R(T, s)^{*} v\right\|^{2} \xi(s, x) d s \\
& =\sum_{\substack{\frac{T-a}{L} \\
L}} \int_{\left\{t \in[0, T] \mid(t, x) \in \overline{\mathcal{T}_{k}(\delta)}\right\}}\left\|B(s)^{*} R(T, s)^{*} v\right\|^{2} \xi(s, x) d s \\
& \geq \sum_{k=0}^{\left\lceil\frac{T-a}{L}\right\rceil} \int_{\left\{t \in[0, T] \mid(t, x) \in \mathcal{T}_{k}(2 \delta)\right\}}\left\|B(s)^{*} R(T, s)^{*} v\right\|^{2} \xi(s, x) d s=v \cdot Q(x, 2 \delta) v .
\end{aligned}
$$

As a result, the positive definiteness of $Q_{x}$ follows from the one of $Q(x, 2 \delta)$.

To conclude, it remains to set, for every $(t, x) \in[0, T] \times[0, L]$,

$$
\eta(t, x) \stackrel{\text { def }}{=}\left\{\begin{array}{cl}
\xi\left(t, \bar{X}^{-1}(t, x)\right) & \text { if }(t, x) \in \mathcal{T}^{\prime} \\
\xi(t, 0) & \text { if } x=\bar{X}(t, 0), \\
0 & \text { if }(t, x) \in \partial([0, T] \times[0, L]) .
\end{array}\right.
$$

Thanks to (A.3d), we have $\eta \in C^{1}([0, T] \times[0, L])$ and (2.11). Moreover,

$$
\eta(t, \bar{X}(t, x))=\xi(t, x), \quad \forall(t, x) \in[0, T] \times[0, L] .
$$

\section{REFERENCES}

[AB03]

[AKBGBdT11]

[BGBPG02]

[CBdN08]
Fatiha Alabau-Boussouira, A two-level energy method for indirect boundary observability and controllability of weakly coupled hyperbolic systems, SIAM J. Control Optim. 42 (2003), no. 3, 871-906 (electronic). MR 2002139 (2004h:93023)

— A hierarchic multi-level energy method for the control of bidiagonal and mixed n-coupled cascade systems of PDE's by a reduced number of controls, Adv. Differential Equations 18 (2013), no. 11-12, 1005-1072. MR 3129017

- Insensitizing exact controls for the scalar wave equation and exact controllability of 2-coupled cascade systems of PDE's by a single control, Math. Control Signals Systems 26 (2014), no. 1, 1-46. MR 3163486

Fatiha Alabau-Boussouira and Matthieu Léautaud, Indirect stabilization of locally coupled wave-type systems, ESAIM Control Optim. Calc. Var. 18 (2012), no. 2, 548-582. MR 2954638

Farid Ammar-Khodja, Assia Benabdallah, Manuel González-Burgos, and Luz de Teresa, Recent results on the controllability of linear coupled parabolic problems: a survey, Math. Control Relat. Fields 1 (2011), no. 3, 267-306. MR 2846087 (2012i:93015)

Olivier Bodart, Manuel González-Burgos, and Rosario Pérez-García, Insensitizing controls for a semilinear heat equation with a superlinear nonlinearity, C. R. Math. Acad. Sci. Paris 335 (2002), no. 8, 677-682. MR 1941648 (2003j:49009) Jean-Michel Coron, Georges Bastin, and Brigitte d'Andréa Novel, Dissipative boundary conditions for one-dimensional nonlinear hyperbolic systems, SIAM J. Control Optim. 47 (2008), no. 3, 1460-1498. MR 2407024 (2009c:93091) 
[CG09a] Jean-Michel Coron and Sergio Guerrero, Local null controllability of the twodimensional Navier-Stokes system in the torus with a control force having a vanishing component, J. Math. Pures Appl. (9) 92 (2009), no. 5, 528-545. MR 2558423 (2010m:93012)

[CG09b] Null controllability of the $N$-dimensional Stokes system with $N-1$ scalar controls, J. Differential Equations 246 (2009), no. 7, 2908-2921. MR 2503028 (2010k:93016)

[CGR10] Jean-Michel Coron, Sergio Guerrero, and Lionel Rosier, Null controllability of a parabolic system with a cubic coupling term, SIAM J. Control Optim. 48 (2010), no. 8, 5629-5653. MR 2745788 (2012b:93017)

[Cir69] Marco Cirinà, Boundary controllability of nonlinear hyperbolic systems, SIAM J. Control 7 (1969), 198-212. MR 0254408 (40 \#7617)

[CL14] Jean-Michel Coron and Pierre Lissy, Local null controllability of the threedimensional Navier-Stokes system with a distributed control having two vanishing components, Invent. Math. 198 (2014), no. 3, 833-880. MR 3279537

[Cor07] Jean-Michel Coron, Control and nonlinearity, Mathematical Surveys and Monographs, vol. 136, American Mathematical Society, Providence, RI, 2007. MR 2302744 (2008d:93001)

[DLRL14] Belhassen Dehman, Jérôme Le Rousseau, and Matthieu Léautaud, Controllability of two coupled wave equations on a compact manifold, Arch. Ration. Mech. Anal. 211 (2014), no. 1, 113-187. MR 3182479

[FCGIP06] Enrique Fernández-Cara, Sergio Guerrero, Oleg Yu. Imanuvilov, and Jean-Pierre Puel, Some controllability results for the $N$-dimensional Navier-Stokes and Boussinesq systems with $N-1$ scalar controls, SIAM J. Control Optim. 45 (2006), no. 1, 146-173 (electronic). MR 2225301 (2007a:93010)

[GBPG05] Manuel González-Burgos and Rosario Pérez-García, Controllability of some coupled parabolic systems by one control force, C. R. Math. Acad. Sci. Paris 340 (2005), no. 2, 125-130. MR 2116769 (2005h:93088)

[Gro86] Mikhael Gromov, Partial differential relations, Ergebnisse der Mathematik und ihrer Grenzgebiete (3) [Results in Mathematics and Related Areas (3)], vol. 9, Springer-Verlag, Berlin, 1986. MR 864505 (90a:58201)

[Gue07] Sergio Guerrero, Null controllability of some systems of two parabolic equations with one control force, SIAM J. Control Optim. 46 (2007), no. 2, 379-394. MR 2309033 (2008h:93014)

[Har82] Philip Hartman, Ordinary differential equations, second ed., Birkhäuser, Boston, Mass., 1982. MR 658490 (83e:34002)

[KHN63] R. E. Kalman, Y. C. Ho, and K. S. Narendra, Controllability of linear dynamical systems, Contributions to Differential Equations 1 (1963), 189-213. MR 0155070 (27 \#5012)

[LJ01] Ta-Tsien Li and Yi Jin, Semi-global $C^{1}$ solution to the mixed initial-boundary value problem for quasilinear hyperbolic systems, Chinese Ann. Math. Ser. B 22 (2001), no. 3, 325-336. MR 1845753 (2002d:35121)

[LR02] Ta-tsien Li and Bopeng Rao, Local exact boundary controllability for a class of quasilinear hyperbolic systems, Chinese Ann. Math. Ser. B 23 (2002), no. 2, 209-218, Dedicated to the memory of Jacques-Louis Lions. MR 1924137 (2003f:93011)

[LR03] Ta-Tsien Li and Bo-Peng Rao, Exact boundary controllability for quasi-linear hyperbolic systems, SIAM J. Control Optim. 41 (2003), no. 6, 1748-1755 (electronic). MR 1972532 (2004b:93016)

[LRW10] Tatsien Li, Bopeng Rao, and Zhiqiang Wang, Exact boundary controllability and observability for first order quasilinear hyperbolic systems with a kind of nonlocal boundary conditions, Discrete Contin. Dyn. Syst. 28 (2010), no. 1, 243257. MR 2629481 (2011m:93019)

[LY85] Ta Tsien Li and Wen Ci Yu, Boundary value problems for quasilinear hyperbolic systems, Duke University Mathematics Series, V, Duke University, Mathematics Department, Durham, NC, 1985. MR 823237 (88g:35115)

[SM67] L. M. Silverman and H. E. Meadows, Controllability and observability in timevariable linear systems, SIAM J. Control 5 (1967), 64-73. MR 0209043 (34 \#8851)

[Wan06] Zhiqiang Wang, Exact controllability for nonautonomous first order quasilinear hyperbolic systems, Chinese Ann. Math. Ser. B 27 (2006), no. 6, 643-656. MR 2273803 (2007h:93022) 
[Zha09] Qi Zhang, Exact boundary controllability with less controls acting on two ends for quasilinear hyperbolic systems, Appl. Math. J. Chinese Univ. Ser. A 24 (2009), no. 1, 65-74. MR 2569281 (2010i:93019) 